\title{
Calculated Neutron Spectrum from 800-MeV Protons Incident on a Copper Beam Stop
}

by

\author{
Dennis G. Perry
}

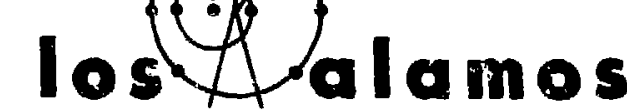

scientifie laboratory

of the Universily of California

los aLAMOS, NEW MEXICO 87545

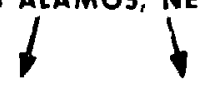

An Alfirnative Retion/Equal Opporiunily Employe:

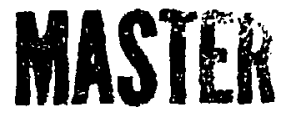


In the interest of prompt distribution, this report was not edited by the Technical Information staff.

Prialed in the Inited Stoten of Amorica. Avarilable trom National Tochnical Information Service

U S Dopartment of Commorce

$$
5285 \text { Pant Roral Road }
$$

$$
\begin{aligned}
& 5285 \text { Port Royal Road } \\
& \text { Springiald, VA } 22151
\end{aligned}
$$

Price: Printed Copt 4.00 Mictofich 52.25

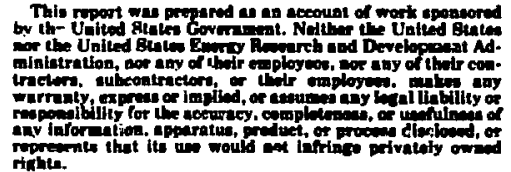




\section{CALCULATED NEUTRON SPECTRUM FROM 800-MeV}

PROTONS INCIDENT ON A COPPER BEAM STOP

by

Dennis G. Perry

\section{ABSTRACT}

A Monte Carlo calculation has been performed to obtain the neutron spectrum generated b 800-MeV protons ilcident on the LAMPF main copper beam stop. The total flux is calculated to be of trs order of $10^{13} \mathrm{n} / \mathrm{cm}^{2}$ - - sec-mA at full-beam intensity of $1 \mathrm{~mA}$, with flux pectra calculated for anglfs of $20^{\circ}, 30^{\circ}$, $60^{\circ}, 90^{\circ}, 10^{\circ}$, and $150^{\circ}$.

\section{INTRODUCTION}

The Clinton P. Anderson Meson Physics Facility (LAMPF) is designed to be a high-intensity itoton accelerator of medium energy $(800 \mathrm{MeV})$. The orimary purpose is to generate mesons of an intel sity not obtainable elsewhere. ${ }^{l}$ Thus, it is primaripy ; tool for the atomir, nuclear, and elementary par. ticle physicist and nuclear chemist, but it als pas important applications in medicine, isotope produc tion, defense science, and in the study of tie structure of materials. Some of these applicat.ons use the primary prcton beam, while others usr. the proton beam as a $\pi$ gans of generating the secorjary particle beam of interest.

One of these secondary reacticirs of interest to the radio and nuclear chemist as well as the materials scientist is the neutron 1 ux generated at the mai, LAMFF proton beam stop. Because the proton neam is expected to reach a.1 intensity of $1 \mathrm{~mA}$, the neutron flux genereced stiuld be one of the most intense available cn a critinuing, long-tern basis anywhere in the world. Because of this incensity, the source of neutruns has attracced interest from the nuclear chemists and materials scientists in various progrars. Because of the intense nestron fluy. expecter in a Controlled Thermonuclear Reactor (CTR), thir source of neutrons can be expected to play

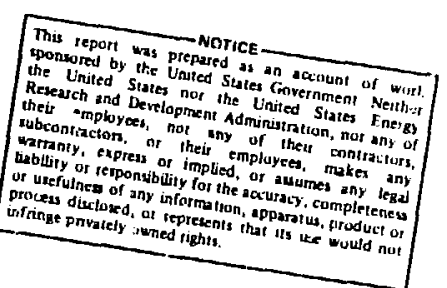

This report was prepuredice

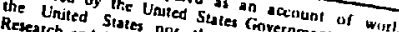
thetr and Development United Stent Neth rubconriactos, not iny of atration, nor Ene'gs

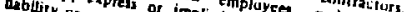
procefulines of anbity for the or atumes any any y rened rights that its ltas

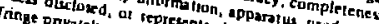

a iarge role in developing materials damage nferfation related to a CTR. But, what intensity can one expect from this source, and what is the spectrum stiape? These questions can be preliminarily answered by theoretical calcuỉations.

\section{I!. CALCULATION}

A Monta Carlo calculation has been performed to estimate the neutron spectrum emerging from the LAMPF Area $A$ beam stop. This calculation gives a predicted spectral shape as well as an expected total intensity. These estimates are useful to those planning experiments at LAMPF, as well as inicial estimates for unfolding the differential neutron pectrum from measured integral foil activities.

The incident proton beam is assumed to have a Gaussian intensity distribution with $95 \%$ of the proto: concained within a radius of $5.7 \mathrm{~cm}{ }^{2}$ The protor inergy incident on the beam stop is assumed to be 75: MeV, allowing $50 \mathrm{MeV}$ of energy loss in the various upstream production targets.

A cross-sectional view of the beam stop configuratica ror these calculations is shown schematically in Fig. 1. Also shown in Fig. 1 is a preumatic tran: fer line (rabbit). The neutrons generated in the cioper beam stop have been transported to the interior of the rabbit and analyzed as a 
TABLE I

DIMENSIONS AND CONPOSITION OF BEAM STOP AREA

\begin{tabular}{|c|c|c|c|c|}
\hline Area & Composition & Boundary & $\begin{array}{l}\text { Radius } \\
(\mathrm{cm})\end{array}$ & $\begin{array}{l}\text { Length } \\
\text { (cm) }\end{array}$ \\
\hline 1 & Cu & 1 & 8.81 & 43.18 \\
\hline 2 & $\mathrm{Cu}+\mathrm{H}_{2} \mathrm{O}$ & 2 & 10.08 & 43.18 \\
\hline 3 & $\mathrm{H}_{2} \mathrm{O}$ & 3 & 10.33 & 43.18 \\
\hline 4 & $\mathrm{Cu}$ & 4 & 10.41 & 43.18 \\
\hline 5 & void & 5 & 11.68 & 205.00 \\
\hline 6 & $\mathrm{Fe}$ & 6 & 90.00 & 205.00 \\
\hline 10 & void & 10 & 3.81 & 137.16 \\
\hline 11 & $\mathrm{Fe}$ & 11 & 3.66 & 137.16 \\
\hline 12 & $\mathrm{H}_{2} \mathrm{O}$ & 12 & 3.98 & 137.16 \\
\hline 13 & $\mathrm{Fe}$ & 13 & 4.13 & 137.16 \\
\hline
\end{tabular}

function of angle with respect to the center of the beam stop. The detailed composition and dimensions of the beam stop and adjacent rabbit are given in Table I, with the numbering system corresponding to that of Fig. 1. The numbers within an area refer to the composition, and the numbers on the liries refer to boundary dimensions. The beam stop may be moved parallel to the rabbit for a total of 66.04 $\mathrm{Cm}$ (see Fig. 2). In the extreme downstream position, the beam stop extends beyond the straight water cooled portion of the rabbit by $3.81 \mathrm{~cm}$. In the extreme upstream position, the straight water cooled porition of the rabbit extends beyond the face of the beam stoo by $31.75 \mathrm{~cm}$.

The Monte Carlo calculations were performed on the Los Alamos Scientific Laboratory (LASL) COC-6600 and $\mathrm{CDC}-7600$ computers using the computer codes NMTC $^{3}$ and MCN. 4 For a previous comparison of experimeital measurements and the output from these codes, see, for example, Ref. 5. The two cades are complimentary to each other in their calculation of the neutron spectrum. The code NATC is used first to generate neutrons of all energies from the proton interaction with the copper bean stop. NMTC then transports to the required toundary those neutrons above some minimum energy, e.g., $20 \mathrm{MeV}$ in these calculations. The code $M C N$ is then used to transport those neutrons below $20 \mathrm{MeV}$ to the same toundary.

The calculations reported here were performed in several steps. In the first step the neutron spectrum was calculated for the surface of the copper beam stop. The second step was to transport the neutrons from the surface of the beam stop to the outer boundary of steel located $90 \mathrm{~cm}$ from the center of the beam stop. Those reutrons which entered the rabbit line were lost to this transport calculation.

The neutrons which entered the rabbit line were then analyzed to obtain the flux as a function of energy and angle with respect to the center of the beam stop. The differential flux, the integral flux, and the flux per unit lethargy are illustrated in Figs. 3-8 for different angles. The angles plotted are defined as the angle between the center of the beam stop and the chosen location in the rabbit line. The flux data of Figs. 3-8 are presented in tabular form in Tabies $I I-V I I I$.

\section{REFERENCES}

1. L. Rosen and D. Nagle, "Introduction to the Proposed Los Alamos Meson Project," Los Alamos Scientific Laboratory report LA-3025-MS.

2. H. Howard, Los Alamos Scientific Laboratory, private commication, 1973.

3. W. A. Coleman and T. W. Armstrong, "The NucieonMeson Transport Code, NMTC," Oak Ridge Nationa? Laboratory report ORNL-46-6 (October 1970).

4. E. D. Cashwell, J. R. Neergaard, W. M. Taylor, and G. D. Turner, "ifCit: A Neutron Monte Carlo Code," Los Alamos Scientific Laboratory report LA-475] (January 1972).

5. R. R. Fullwood, J. D. Cramer, R. A. Haarman, R. P. Forrest, Jr., and R. G. Schrandt, "Neutron Production by Medium-Energy Protons on Heavy Metal Targets," Los Alamos Scientific Laboratory report LA-4789 (January 1972). 


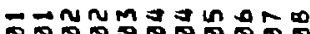

s. 에을 zom

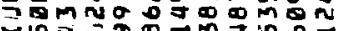

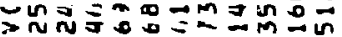

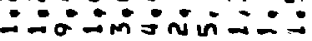

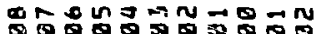

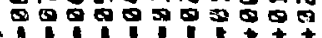

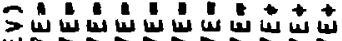

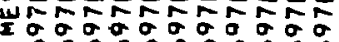

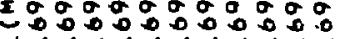

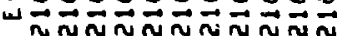

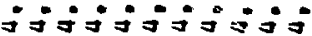

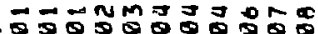
is: •

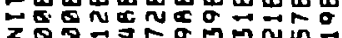
zom $\Rightarrow$

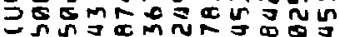

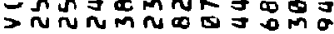
$\therefore-\div$ in in $0 \div-\div$ i

ราง

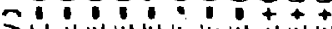

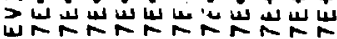

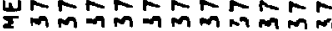

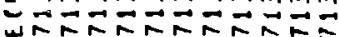
Mmmm mM mM niñnininininit

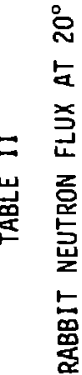

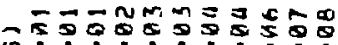
की

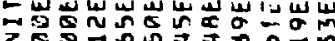

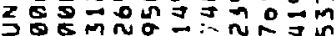

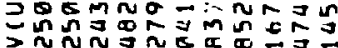

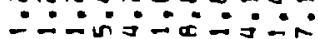

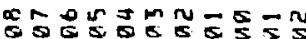
万.
2010

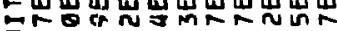

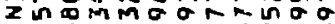
$30 M m m$ in in $\infty \pi N$. $\sim M N \rightarrow N=0 m \geqslant \infty$ $>n: M E a m 00 m \rightarrow J$ $\therefore$ -

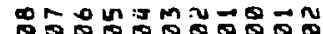

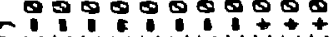

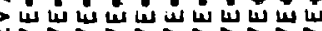
L

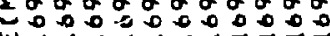

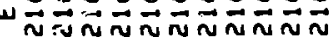

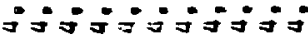

on a 1000

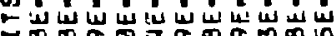

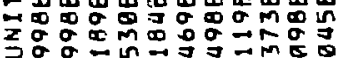
马o

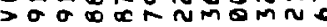

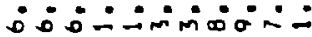

व

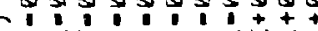

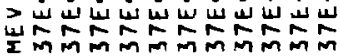
$=\vec{H}=\vec{D} \vec{R} \vec{R} \vec{R} \vec{M}$ $m$ m $M m M M$ in $m$ inininininiñ

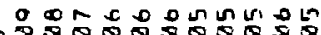

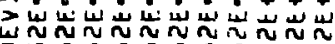
I UMm MmMMmM

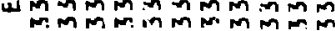
$\because \div \div \div \div: \div$

-

क

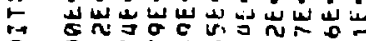

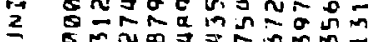

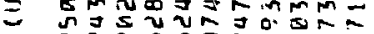

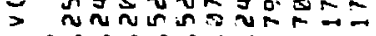

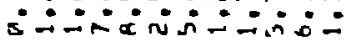

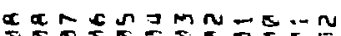
15055:55:55e

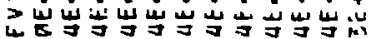

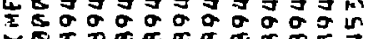

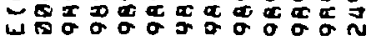

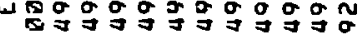

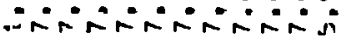

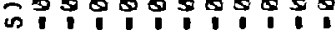

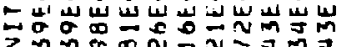

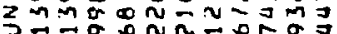

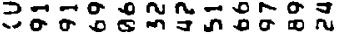

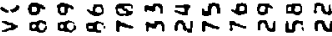

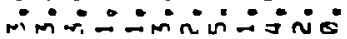

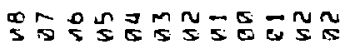

1.11:11 1 1 + +

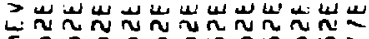

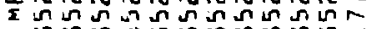
-Mmm $m m m m m m F$

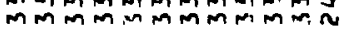

$\because \therefore \therefore \div \div \div \div \div \div \div 0$

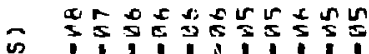

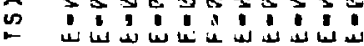

z o

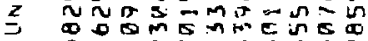

$\exists \quad x$

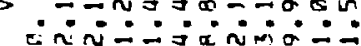

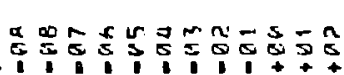

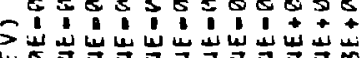

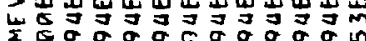

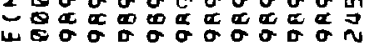

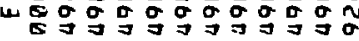

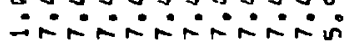

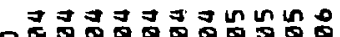
का: 七崖岁岁出出出 zomomomm

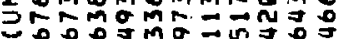

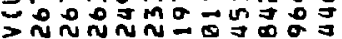

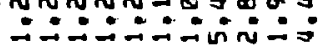

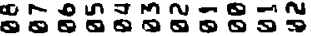
-1. 1. >

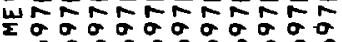
40000000000 $\vec{n} \vec{n} \vec{N} \vec{n} \vec{n} \vec{n} \vec{n} \vec{n} \vec{n} \vec{N} \vec{N}$ जः:

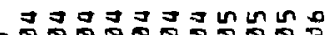

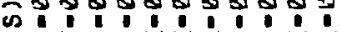

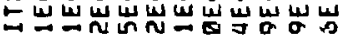

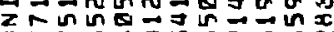

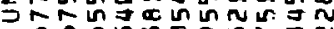
\& 0 Onmsonn บกบN.

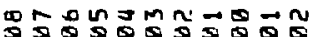

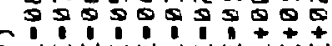
>

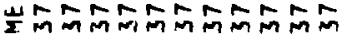

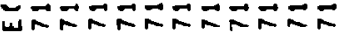
mmmmmm mm inininininin

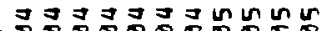

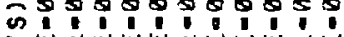

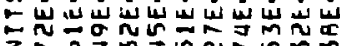

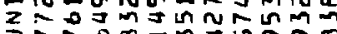

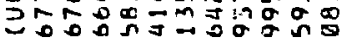

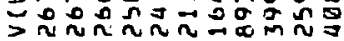

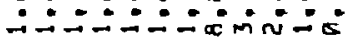

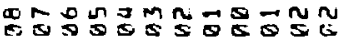
-

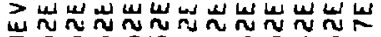

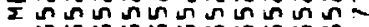
UmmMMmMMm

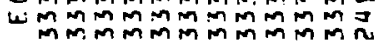

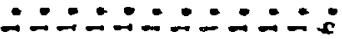

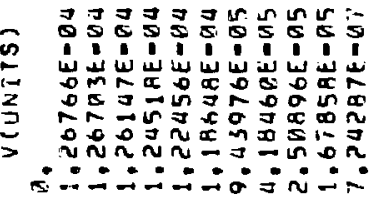

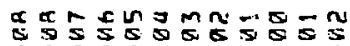
$=1.51100$

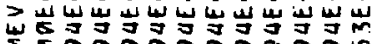

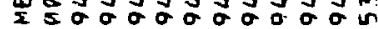

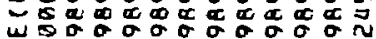

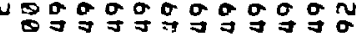

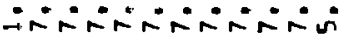


DIFFERENTIAL FLIJX, UNITS= NEUTRONS/CMHEZ-HEV-P

\begin{tabular}{|c|c|c|c|}
\hline 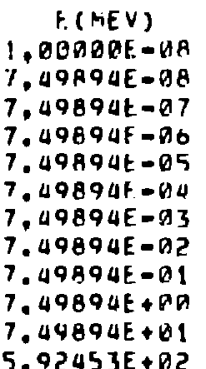 & $\begin{array}{l}V(U N I T S) \\
3 . \\
5.00000 E-01 \\
3.30000 E-01 \\
7.65175 E-02 \\
1.91324 E-02 \\
2.38145 E-03 \\
2.35099 E-03 \\
3.26649 E-04 \\
9.69442 E-05 \\
3.56627 E-06 \\
1.73385 E-07 \\
1.29403 E-08\end{array}$ & $\begin{array}{c}E(M E V) \\
1.33352 E-08 \\
1.33352 E-Q 7 \\
1.33352 E-06 \\
1.33352 E-05 \\
1.33352 E-\theta 4 \\
1.33352 E-03 \\
1.33352 E-02 \\
1.33352 E-\theta 1 \\
1.33352 E+00 \\
1.33352 E+\theta 1 \\
1.33352 F+\theta 2\end{array}$ & $\begin{array}{c}V(U N I T S) \\
5.00000 E-91 \\
5,09000 E-01 \\
3.00000 E-01 \\
8,00952 E-02 \\
1.91526 E=02 \\
3,96839 E-03 \\
1.51462 E-03 \\
3.85380 E-04 \\
5.95457 E-05 \\
5.46442 E-07 \\
6.78233 E-08\end{array}$ \\
\hline
\end{tabular}

FLUX PER UNIT LFTHARGY, UNITS = NEUTRONS/CM\#\# P-P

\begin{tabular}{|c|}
\hline 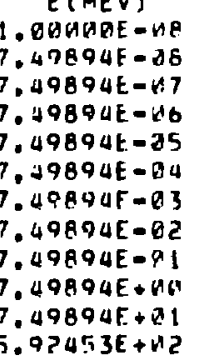 \\
\hline
\end{tabular}

V(UNTTS)

3.

8. $73317 E-B \theta$

$0.12722 E=07$

$1.37455 E-06$

$3.34938 E-\Delta 6$

$4.169(1) 4 E-86$

$4.11571 E-815$

$5.71841 F-05$

$1.69713 \mathrm{E}-314$

$6.243216-15$

$3.03533 t-65$
E (MFV)

. $33352 t=08$ $1.33352 E=08$
$1.33352 E-07$

1. $33352 E-B 6$

i. $33352 E-85$

$1.33352 E-04$

$1.33352 E-03$

$1.33352 E-92$

1.3335 2E-PI

$1.33352 E+19$

$1,33332 E+01$

$1.33352 E+$ HC

H. $24177 E+O$ ?

INTEGRAL FLUX, UAITS = HFUTHOAS/CM\#\#?-P

\begin{tabular}{|c|c|}
\hline 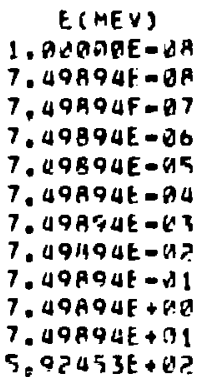 & 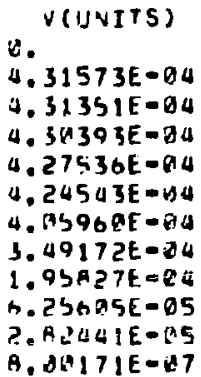 \\
\hline
\end{tabular}

V(UNITS) $1.55656 E-108$ $1.556 \zeta 6 E-07$ $9.33935 E-07$ $2.74251 E-D 6$ $\$ .16002 E-06$ $1.23541 \mathrm{t}-05$ $4.71520 E-05$ $1.19973 \mathrm{E}-84$ $1.85373 E=04$ $1.70114 E=05$ 2. 11142E-OS a.

V(UNITS) 4. $31590 E-84$ 4.31551E-BA 4.3119BE-BA 4. 30649E-BS $4.26699 E-00$ $4.23501 E-Q 0$ $3.95670 \mathrm{E}=04$ 3.95670E-O $1.53347 E-9$ $4.69525 E-25$ 2. $10557 \mathrm{E}-05$ c. - 34875E-
E. (MEV) $2.37137 \mathrm{E}-08$ $2,37137 \mathrm{E}-07$ $2,37137 E-96$ $2,37137 E=06$
$2,31137 E=05$ $2.31137 E=05$ $2.37137 E-44$ $2.37137 E=03$ $2,37137 \mathrm{E}-0 \mathrm{C}$ $2,37137 E-01$ $2,37137 E+\Delta \theta$ $2.37157 E+\theta 1$ 2.37137E+Q2

E (MEV) $2.37137 E-68$ 2. $37137 E=07$ $2.37137 \mathrm{E}-106$ $2.37137 \mathrm{E}-05$ ?.37137E-BA ?. $37137 E-\theta 3$ $2,37137 t-02$ $2,37137 E-B 1$ 2. $37131 \mathrm{E}+\forall$ 2. $371375+\theta$ $2.37137 E+0$ $2.37137 E+112$

E (MEV) $2.37137 E=08$ $2.371371-07$ $2.37137 E-96$ $2.3713 T E-45$ $P, 37137 \mathrm{E}=04$ $2.37137 t-03$ $2.37137 E-12$ 2. $3137 \mathrm{E}-\mathrm{O}$ 2.37 i $75+60$ C. 37137E+41 $? .37137 E+61$ ?.37137E+U? $\checkmark$ (UNITS) 5. $00000 E-01$ 4. 5B000E-O $2.22960 E=01$ . $79434 E=0$ $5.22556 E-03$ $4,45304 E-03$ $1.24437 E-63$ $3,62062 E=04$ $9.81996 E-06$ $3.95557 E-07$ 3.05707E-68 $2,76799 E-08$ 2. $2119 E-07$ $1.25430 E-0$ $3.76133 t=0$ $2,89286 E=0$ $2.46519 E=05$ 6. 8B8BGE-05 $2.00436 E-84$ $5.43630 E-85$ $2.19189 E-05$ $1.69238 E=05$

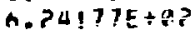

$V$ (UNITS) $4.31592 E-04$ $4.31512 E-04$ $4.30965 E-04$ $4,293645-64$ $4,25909 E-B 4$ $4.20413 E-04$ 3. B 3883E- ba 3. D48BUE-6A 1. $7056 E-94$ $4.26993 E-05$ $! 53772 E-95$
E (MEV) 4. $21697 E-68$ 4. $21697 E=07$ $4.21697 E-86$ 4. $21697 E-05$ $4,21697 \mathrm{E}=04$ $4.2 ! 697 E-O S$ H. $21697 E-42$ $4,21697 E-81$ $4.21697 E+00$ $4.21697 E+B 1$ $4.21697 E+02$ $\checkmark$ (UNITS) 5,00000E-D $4,00000 \mathrm{E}-1 \mathrm{I}$ $1.86964 \mathrm{E}=\mathrm{BI}$ $3.0696 E D$ $3.60544 E-02$ $2.60894 E=03$ . $36020 E$ DB3 $1.10590 \mathrm{E}=04$ $2,39511 E=04$ $1.25573 E=05$ $3,64686 E=07$ $4,20376 E=08$

E (MEV) $4.21697 E-0 B$ $4.21697 \mathrm{E}-0 \mathrm{O}$ 4. $16975-05$ 4. $21697 \mathrm{E}-85$ $4: 21697 E-04$ a. $21697 \mathrm{E}-63$ $4.21697 E-02$ $4.21697 E-0 !$ 4. $21697 E+00$ $4.21697 \mathrm{E}+\Delta !$ $4.21697 E+82$

E ( AEY) 4. 21697E-00 (67E-67 $4.21697 E-06$ $1.21697 \mathrm{E}-05$ $4.21697 \mathrm{E}-04$ $4.21697 E=03$ $4.21697 E-02$ $4.21697 E-D 1$ $4.21697 E+\theta 0$ 4. 1647506 4. $21697 \mathrm{E}+92$
V (UNITS) $4.92228 E=68$ $3.93782 E=67$ $1.053015=06$ $3,54940 E=06$ $2,56834 \mathrm{E}$ at $3.31585 E-05$ $6.90546 F-05$ $2.35709 t=04$ $1,23621 L-04$ $3.59018 E-65$ $4.13843 E=05$

V(UNITS) $4.31585 t-04$ 4. $3145 B E-O A$ 4.30656E-RA $4.20423 E-04$ $4.25185 E=04$ 4. $14249 E-04$ $2.54772 E-64$ 9. $34648 E-85$ $3.72194 E-05$ $1.11462 E-85$ $3.66660 E-84$ 


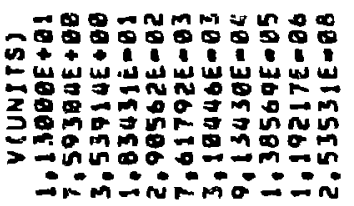

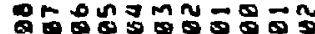

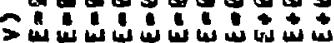

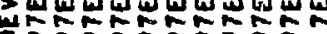

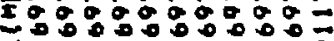
แ $\Rightarrow 050 ; 0050$ in

$0+*+1$. -

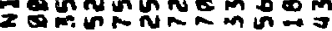
$300 m g$ in 0 in

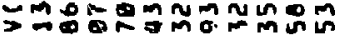

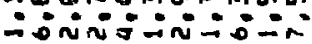

ริำ S

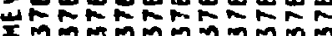
U iñunininininis

응 $\infty 98595000 \%$ —

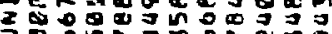
200 m $-m a n=0$

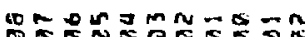

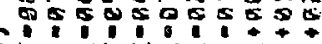

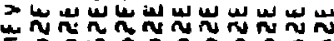
In L $m m m m m m m$

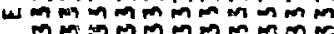
$\div \div \div \div \div \div \div \div \div-$

- ED

$\Rightarrow \quad 00150005$

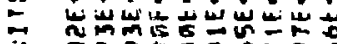

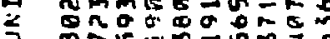

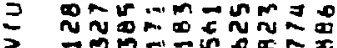
i inivinivi

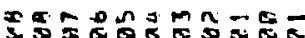

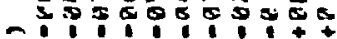

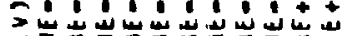

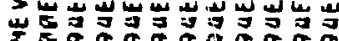
< 60 \%

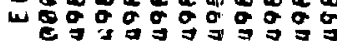
-rimirarirar

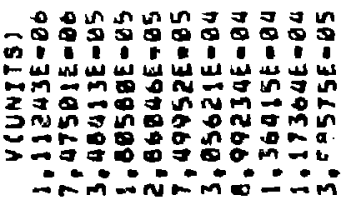

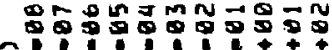

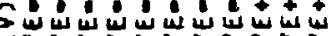

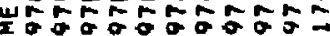
2099090900900

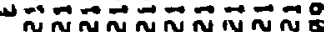

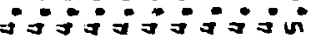

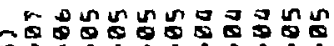
की 0 \% : ว

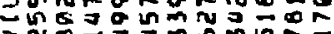
$N \infty \Rightarrow \exists m$ m nn $m$

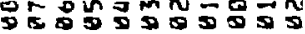
O4

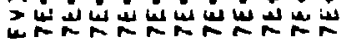

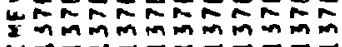

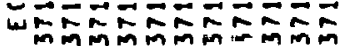
ninininininin

roc un un m ต

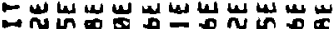

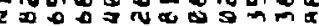
So

$>$ in $\rightarrow$ mo $\rightarrow \infty N R=1$.

जiñ-inicimis:

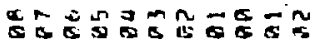
S1 1

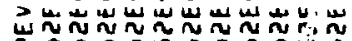

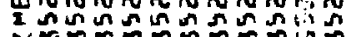

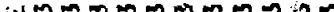

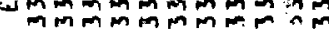
$\therefore \therefore \div \therefore \div=\div \div \div \div$

c c v $15 \% 500 \%$

-

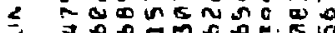

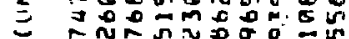
$=\pi N R N D \sigma 0 \leq 4$

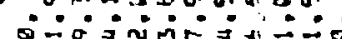

a - 595550056550

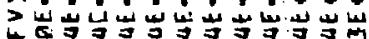

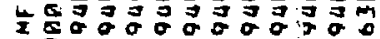

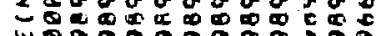

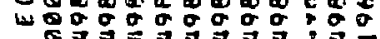

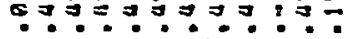

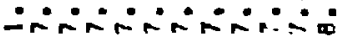

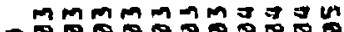

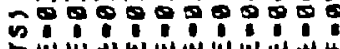

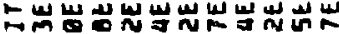
Zms. 9980

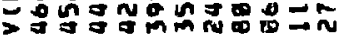

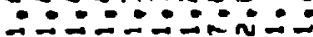

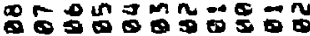
- 4 Dww

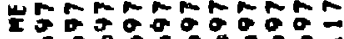
$=0000000000$

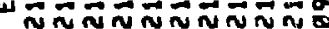

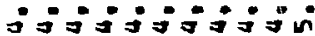

M Mmmm क orog in m o 0

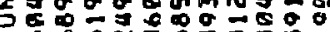

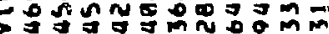
$\because-2 \div=-0 \times 1 n$

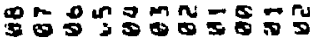
כ

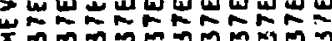

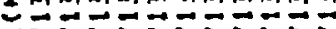

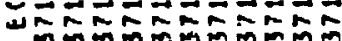

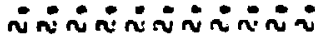

$m m m m m m m \exists M$ $\Leftrightarrow$ \% : ○

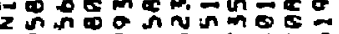
3 o o $0=m=n$ in

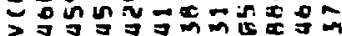
$\therefore \therefore-\div-1=-i=$

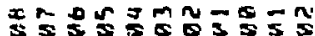
$-555500355$

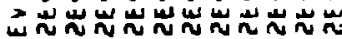
Iñ u.

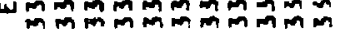

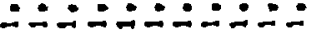

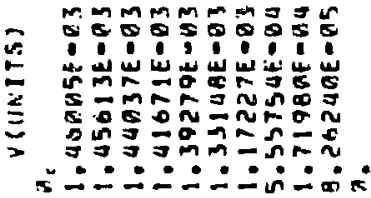

a d 0 L $-56505650505$

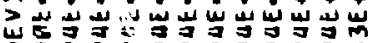

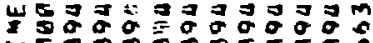

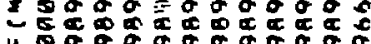
$469 \% \sigma \sigma o \sigma o \sigma o \sigma$ -rarararám 


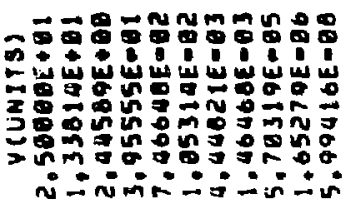

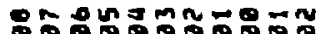
- 9800890000

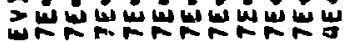

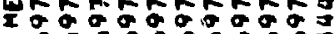
- 0900090000

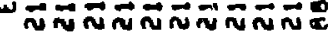

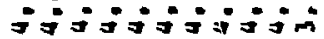

क

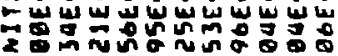
$56 n \mathrm{~m} \rightarrow \mathrm{m}$ in us 0

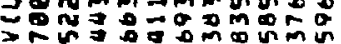

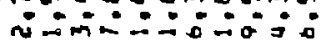

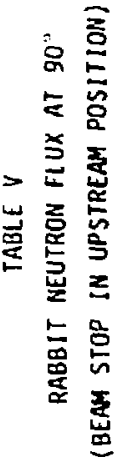

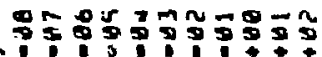

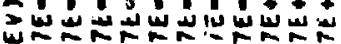

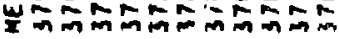

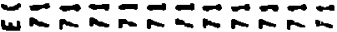

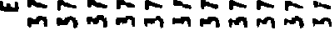
vininivininis

-

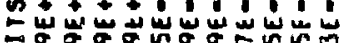

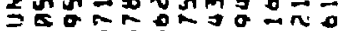

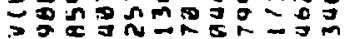

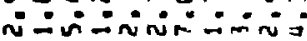

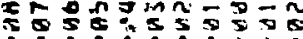
万́

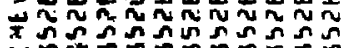

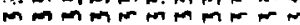
$\because \because \because \because \because \div \therefore \therefore$

Fesconmon

in sos

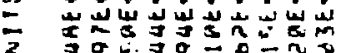

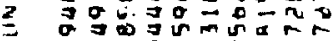

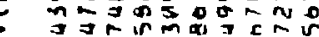

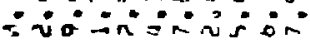

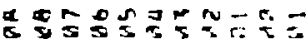
-

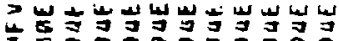

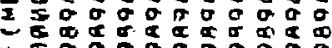

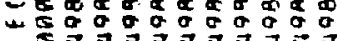

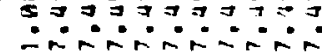

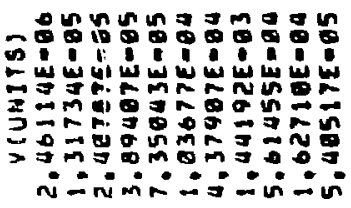

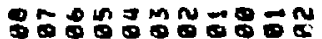

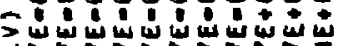

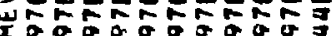
工

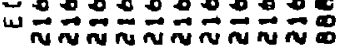
$\because 0^{\circ}=50^{\circ}$

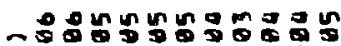
n 造出出㟧。

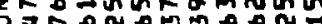

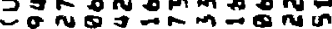

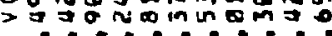

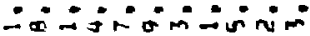

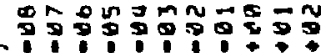

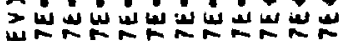

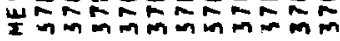

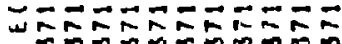
जunisinisin

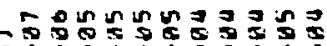
क 191.10

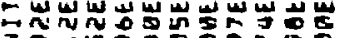
г 万o

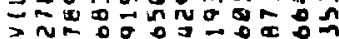
on

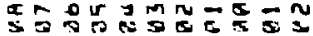
1. 11.10

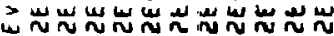

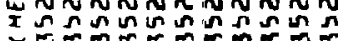
$+\rightarrow n m a m n=m$

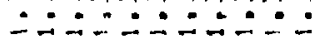

-

구의

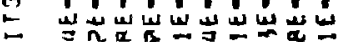

$>$ o

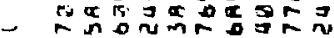

$>$ notuvingond

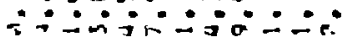

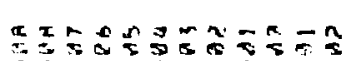

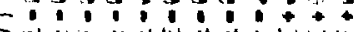

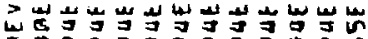

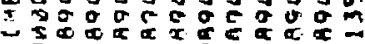

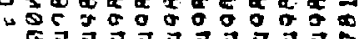

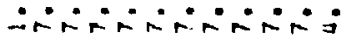

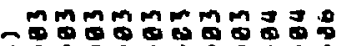
की

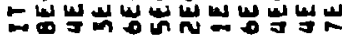

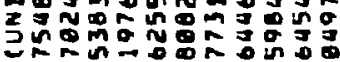

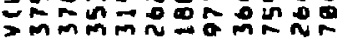

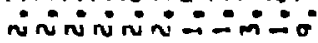

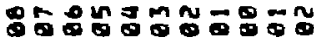
19.19

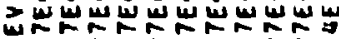
र

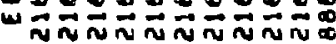
व:5950

mmmmmmg

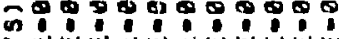

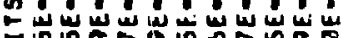
วทñans Nㅡำ mmmnn: ininininin:-:

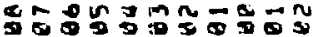
1. 1: : : : >

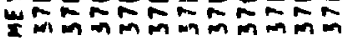

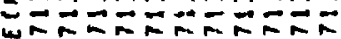
momming om mon

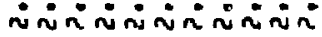

mmmmmmm

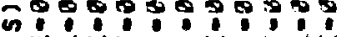

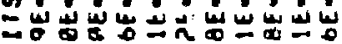
ว ว 0m

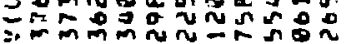
ninininivinis

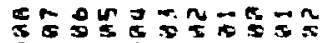

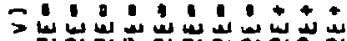

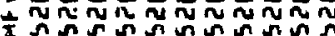

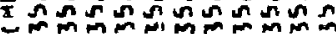
एँ

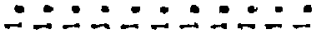

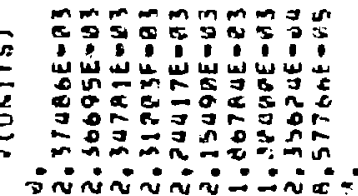

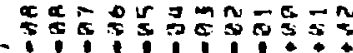

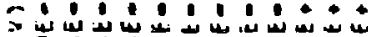

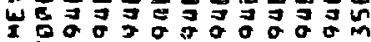
I

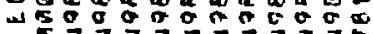
$\therefore r+a r a r a n$ 


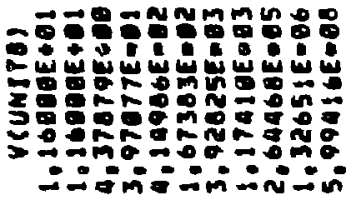

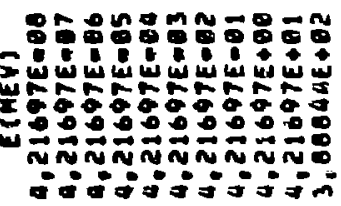

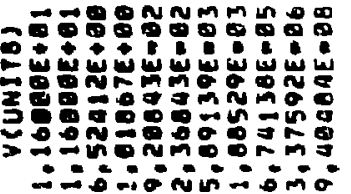

969.

$-8960 \% 006$

2мш

w

$\rightarrow \rightarrow \infty \rightarrow+\infty \rightarrow \infty-1$

W

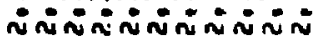

$\leftleftarrows \delta$

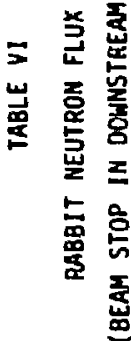

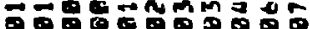

$6+400 \%$

-

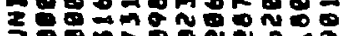

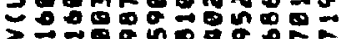

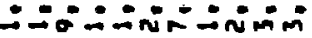

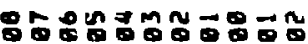

-

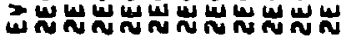
In $x$ un uns

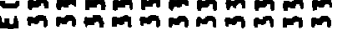

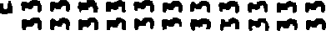

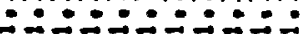

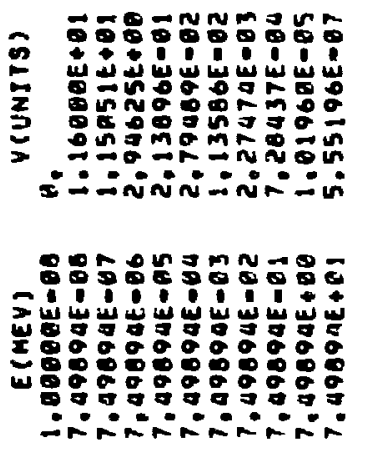

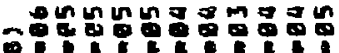
zo. Za $=90$ in $\rightarrow \because 000$

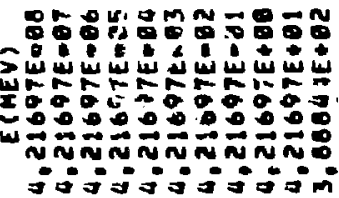

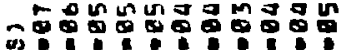

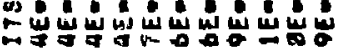

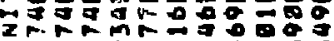
วHRM

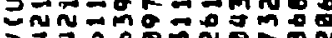
อ

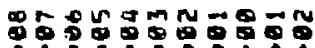
$-90809000$ >

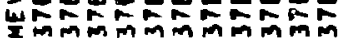

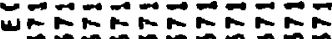
mmmm

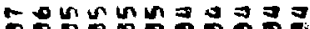
की 000000000 ow.

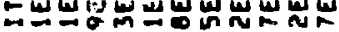

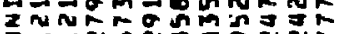

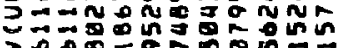

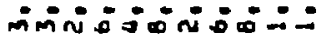

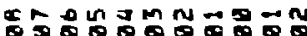
-

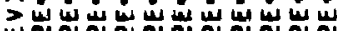

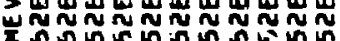

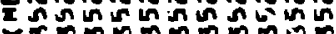
L

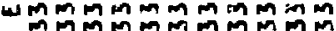

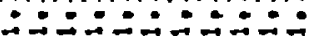

- Dun

is: : :

-

z

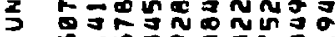

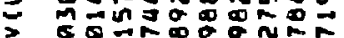

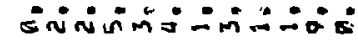

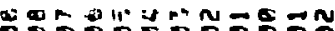
\$0000500006\%

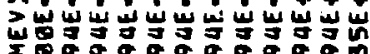

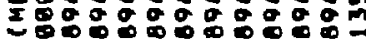
ш 9.

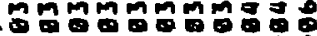
क -

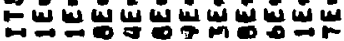

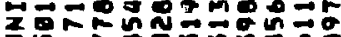
Sn $=$ a

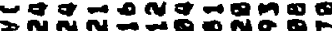

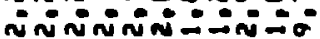

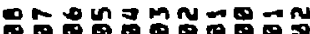
\%日9080005

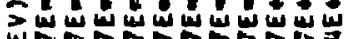

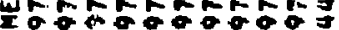

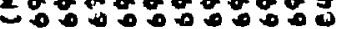
U $\rightarrow \rightarrow \rightarrow \rightarrow \rightarrow \infty$ N N N N N N N ND $\Rightarrow 0=0 ; 00 ; 00$ M

m m mmmmg

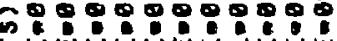
-

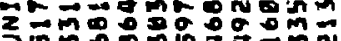
sum -

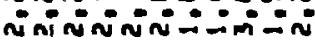

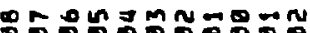
- 100809000 w

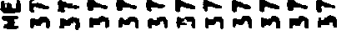

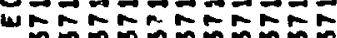

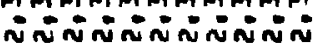

$m m m m M g$ 6\%8. -

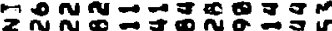
Zñ

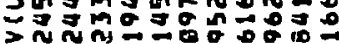

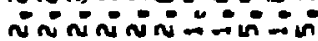

on日 $3 m N=0-N$ -

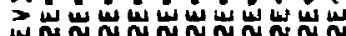

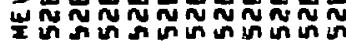
in $\Omega$ un nn un un in ज $m$ m $m$ m $m M m M$ m?

mmmmmmn:z

क 900000005 - w w $\geq \quad$ Norog

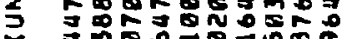

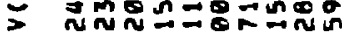

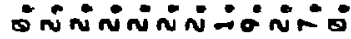

क⿻上丨

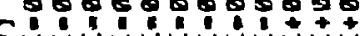
$>\mu \omega \omega \omega \dot{\omega} \omega \omega \omega \omega \omega$

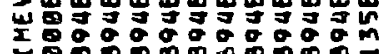

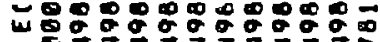

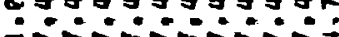




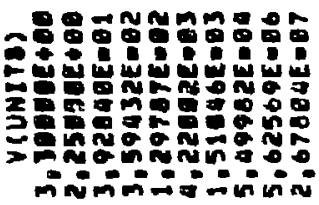

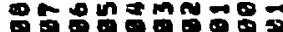
>

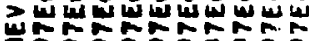

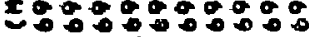
490009010

nennn nn nn $-\Rightarrow 00000000$

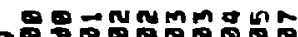

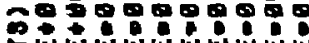

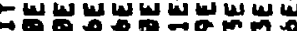

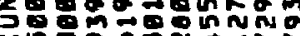

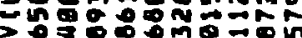
matm

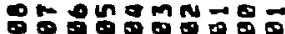
ก:9

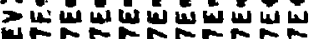

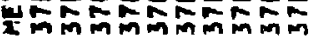
$\because \Rightarrow \Rightarrow+\infty \Rightarrow$ M พ

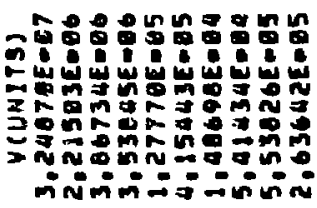

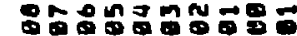

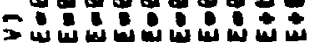

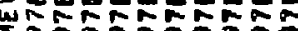

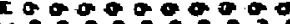

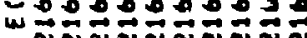
N N N N N N n N

-

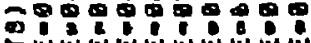

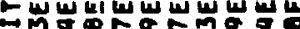
$700=8$ 568

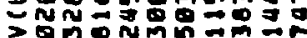
n क5

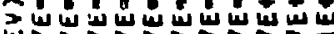
W $\rightarrow \vec{x} \rightarrow-\infty \rightarrow \infty$ MAM M M M m m ผ

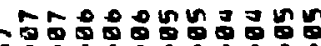

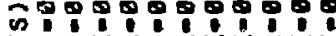
レ

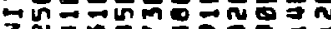
ZN寸MOR

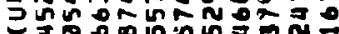
-山山

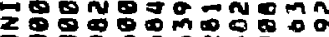

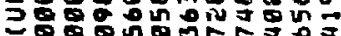

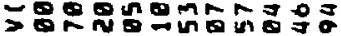
$\Rightarrow N \rightarrow n+\infty=$

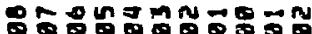
- 0000000000

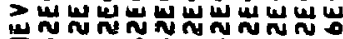

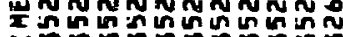

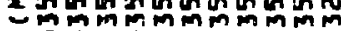
$\omega m m m m i m m m$ in

$\because=1 \div \div \div=1$.

OOHNNm

as $\quad$ 4003\%

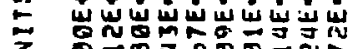

2 \$

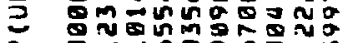

-

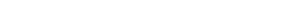

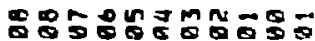
- 0050800905

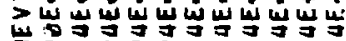

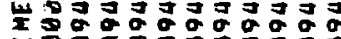

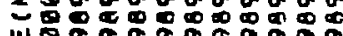

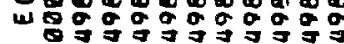
$\Rightarrow R M R R M R R$

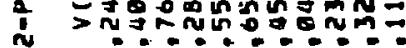
- Ominos óninin

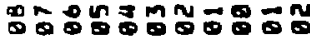
- : : 1.1.+.

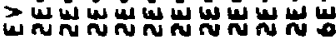

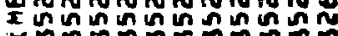
然 mmmmimm m $\because=-\div=\div \div \div=$

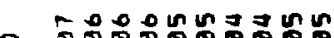

$\rightarrow$ i $19 \%$ :

-

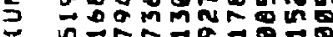

$=n=R-70-0=0$ sunmàñmm

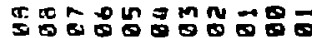
-1.1.1.1.

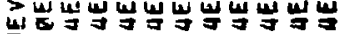

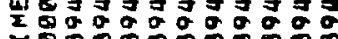
ㄴ.

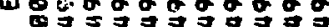

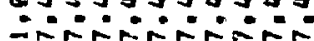

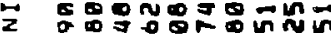

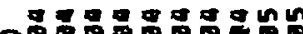

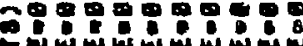

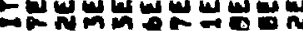
200-00\%

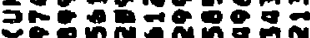
$>-9-60000$ $-1-i=0.00$
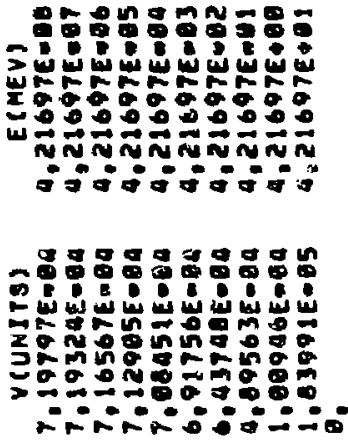

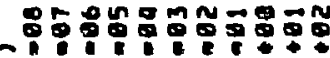
>

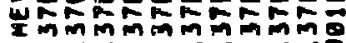
$\rightarrow-2 \rightarrow-2=0$ ตnलm

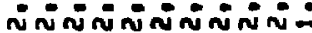

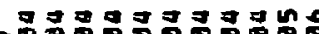

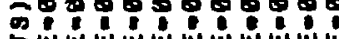

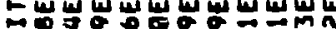

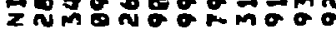
วon in

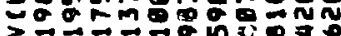

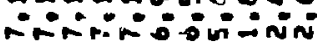

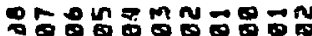

a $21.18 \%$

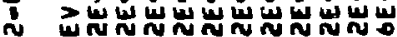

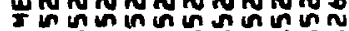
in

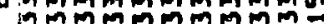
$\therefore \approx \div \div \div \div \div \div=$

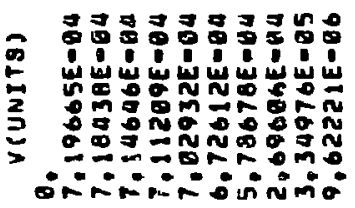

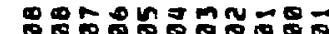
c日. 1.

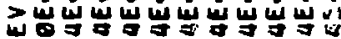

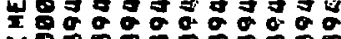

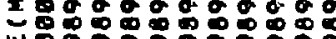

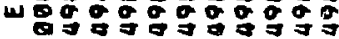
$\rightarrow \pi r a r a r a r$ 


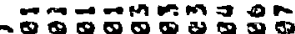

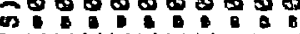

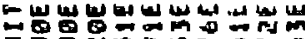
200 Tumonoun 3.

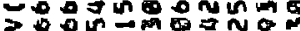
- $=0$ m.

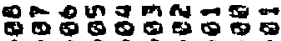

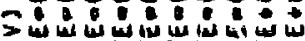

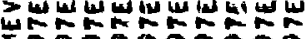
100000000\% -0.0000 13000

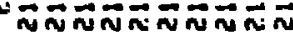

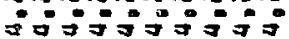

60.0\%:

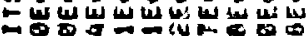

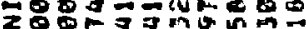
50두은

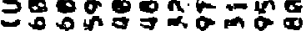
0 60 nn $\because=\div$ : $=0$

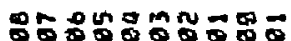

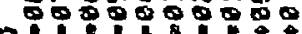

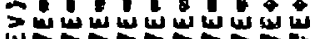

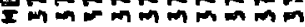
UЕЕ mmmmmm

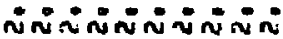

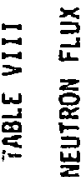

ำ

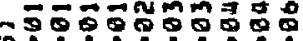
o

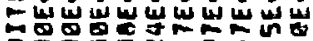

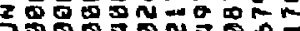

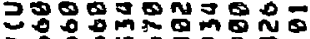
$>0 \% 0=30090$ $\because ே ニ=$ miña:

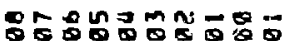
-

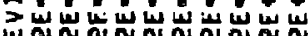
I in in in Umm m m m m m

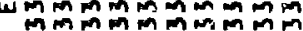
$\therefore \div \div \div$

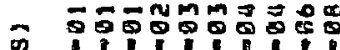

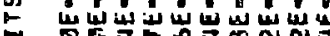

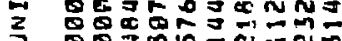

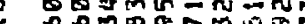

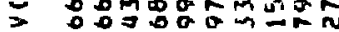

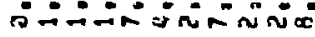

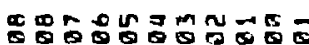
1::1,1.1.+ > 山ل

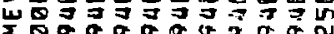

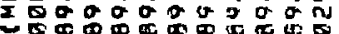

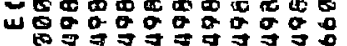
annarian

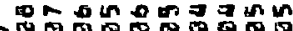

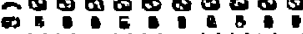

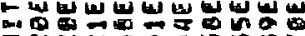
ZNNNMEOUM 30 \%

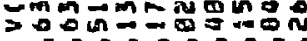
$=-1000=0$ in

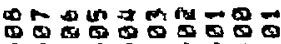
布

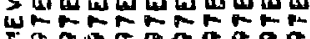
山 505:

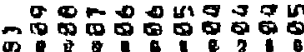

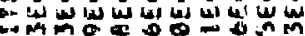
nmocos

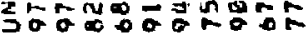
วo: $>-00$ orm $\because \div ; 0$ -

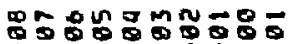
3 HNFF

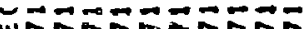
Un NAN

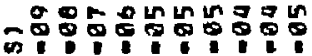

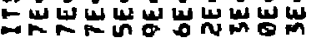
zNM วด:

\section{.}

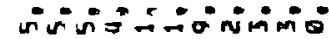

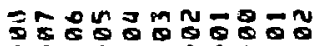

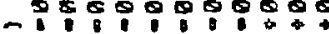
ح

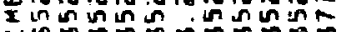

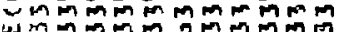
ม $\mathrm{m}$ लिभm $\because \div \div \div$.

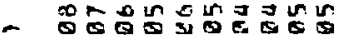

क 010

—

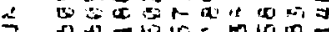

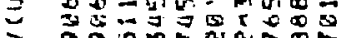

sniñ

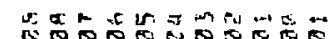
- To8

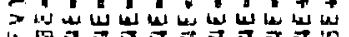
2.

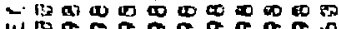

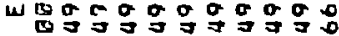
$\rightarrow \pi n-a m a n a$

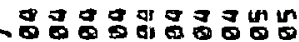

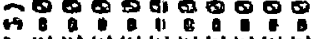

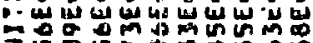

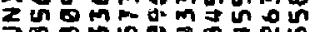
200mud

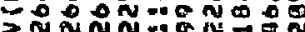
$\therefore$

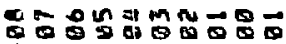
$=6$. 岕出出出出出出

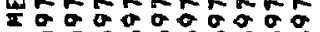
$=0000000000$

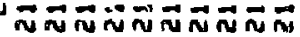

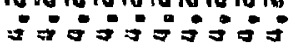

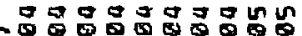
\%

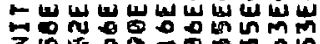

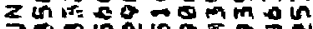
马o: uñ

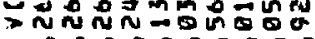
ririna unos

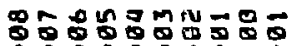
-

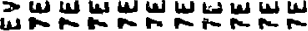
T的的的的的向 UR mmмmmmmm บ

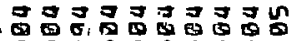
os

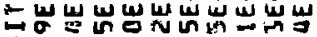

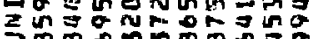

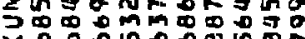

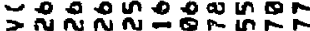
nnñán-

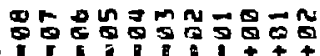

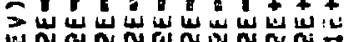

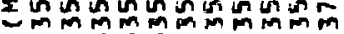

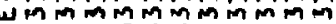

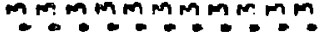

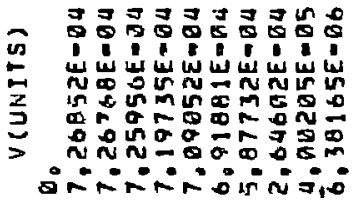

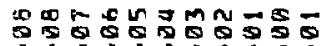
ᄃ

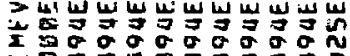

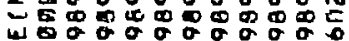

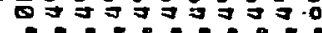

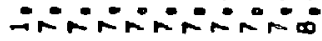




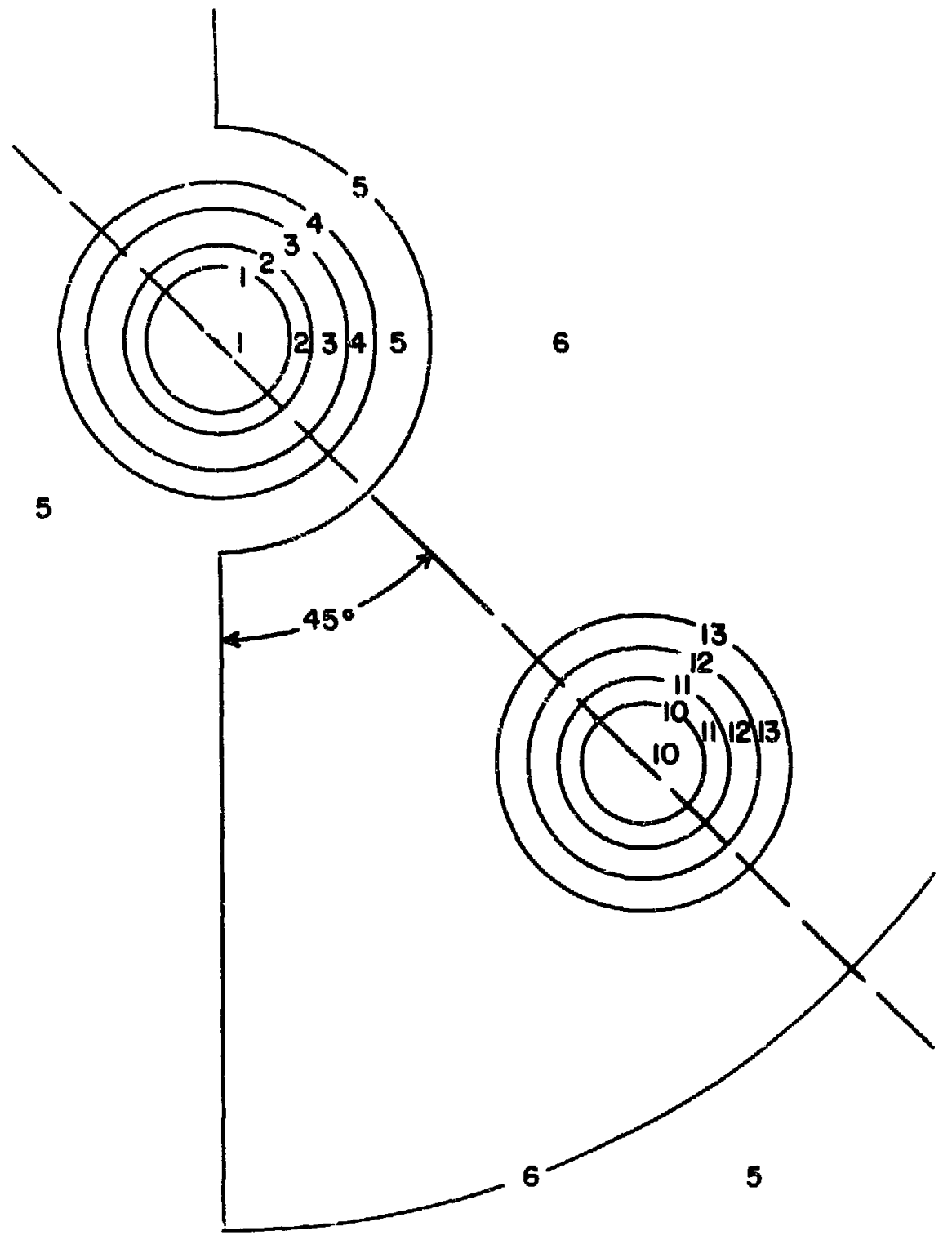

Fig. 1. Cross-sectional view of the beam stop and rabbit configuration. The areas and bouridary numbers correspond to those of Table $I$. 


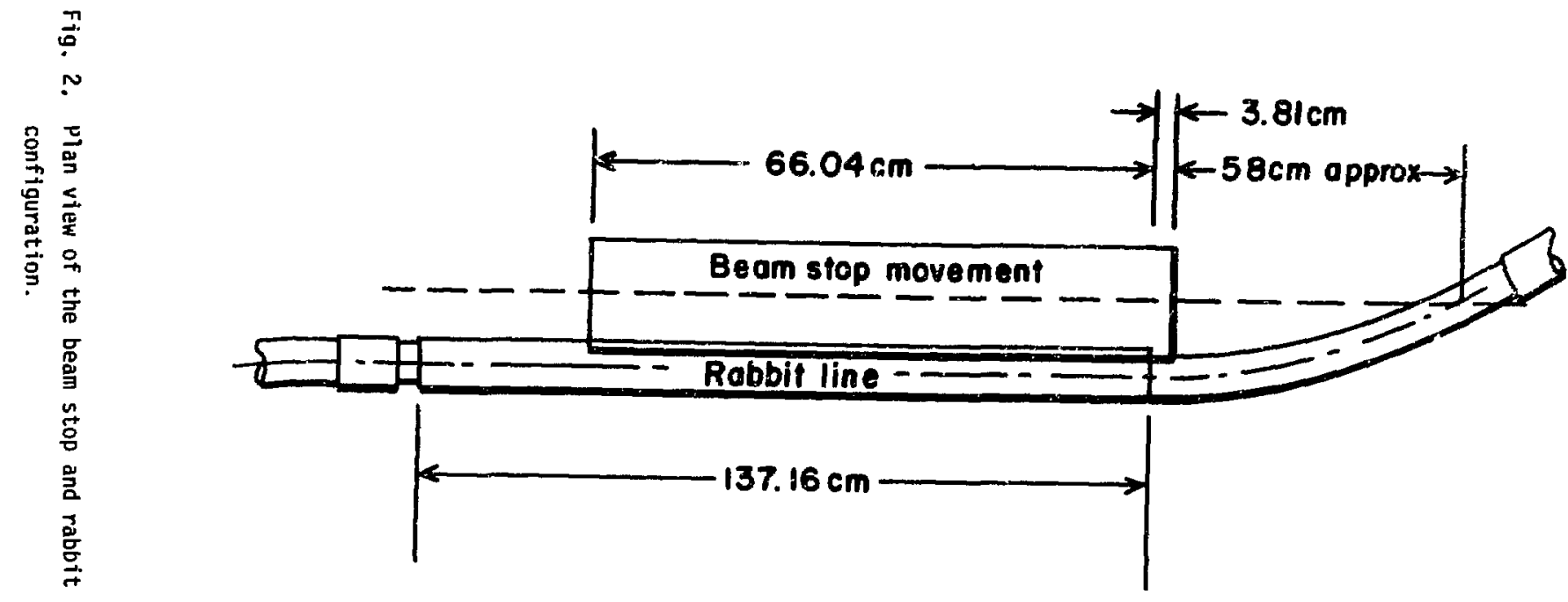

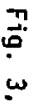

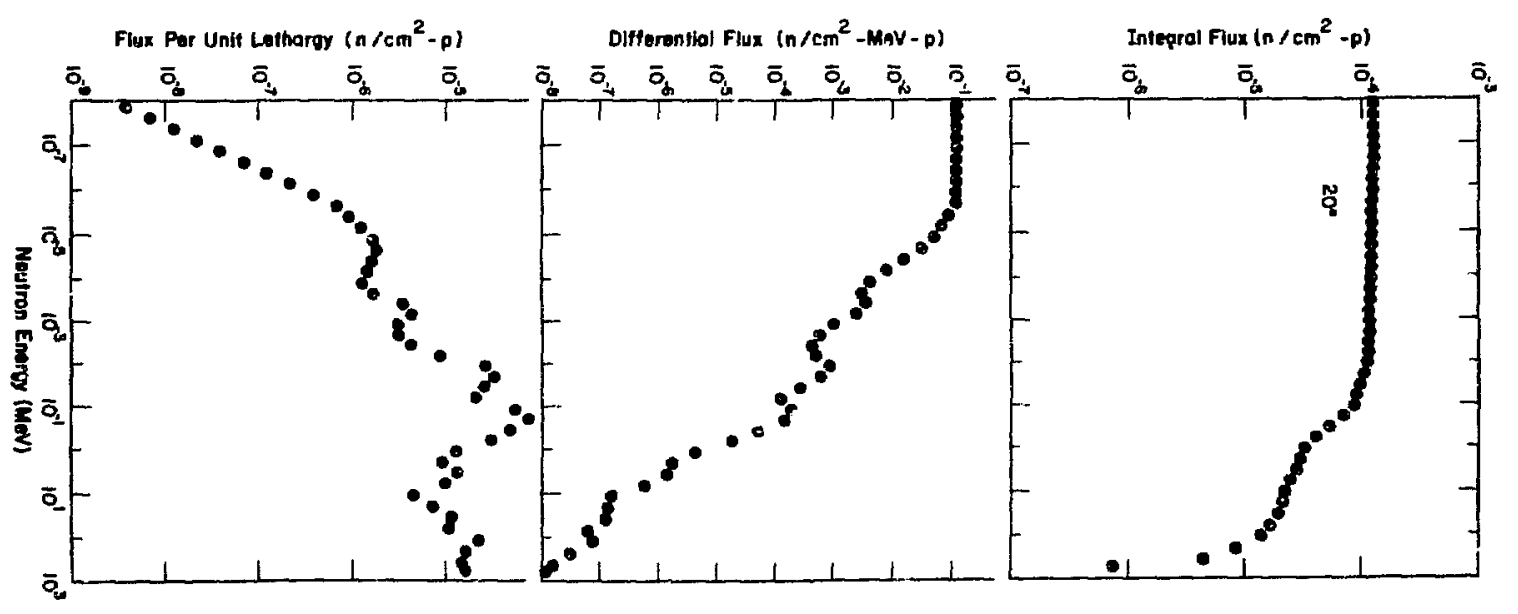




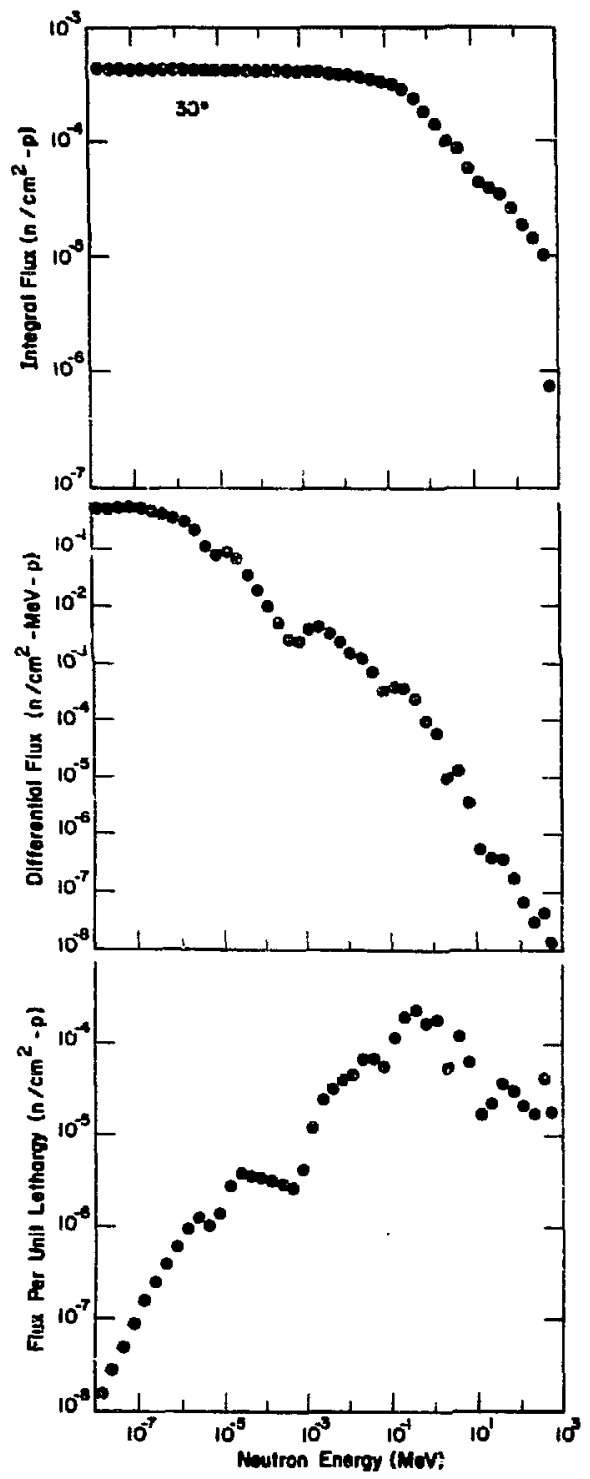

Fig. 4. Calculated neutron flux in the rabbit iine for an angle of $30^{\circ}$ from the center of the copper beam stop.

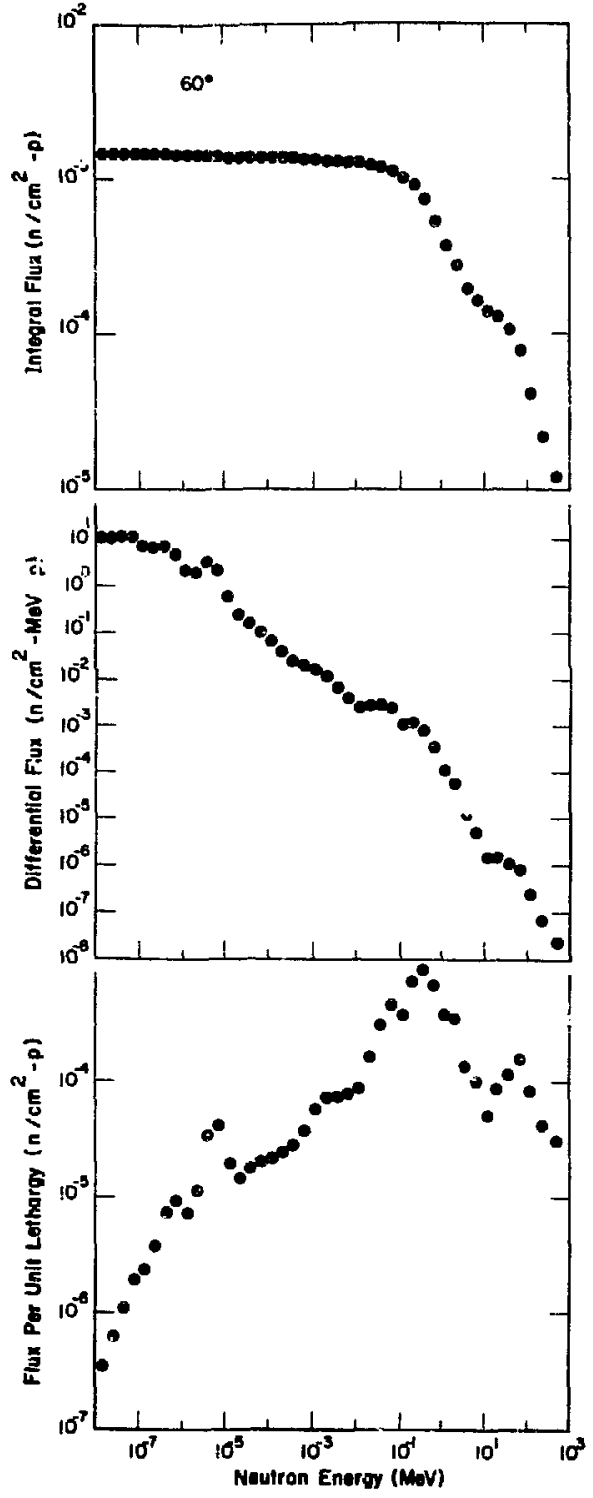

Fig. 5. Calculated neutron flux in the rabbit line for an angle of $60^{\circ}$ from the center of the copper beam stop. 

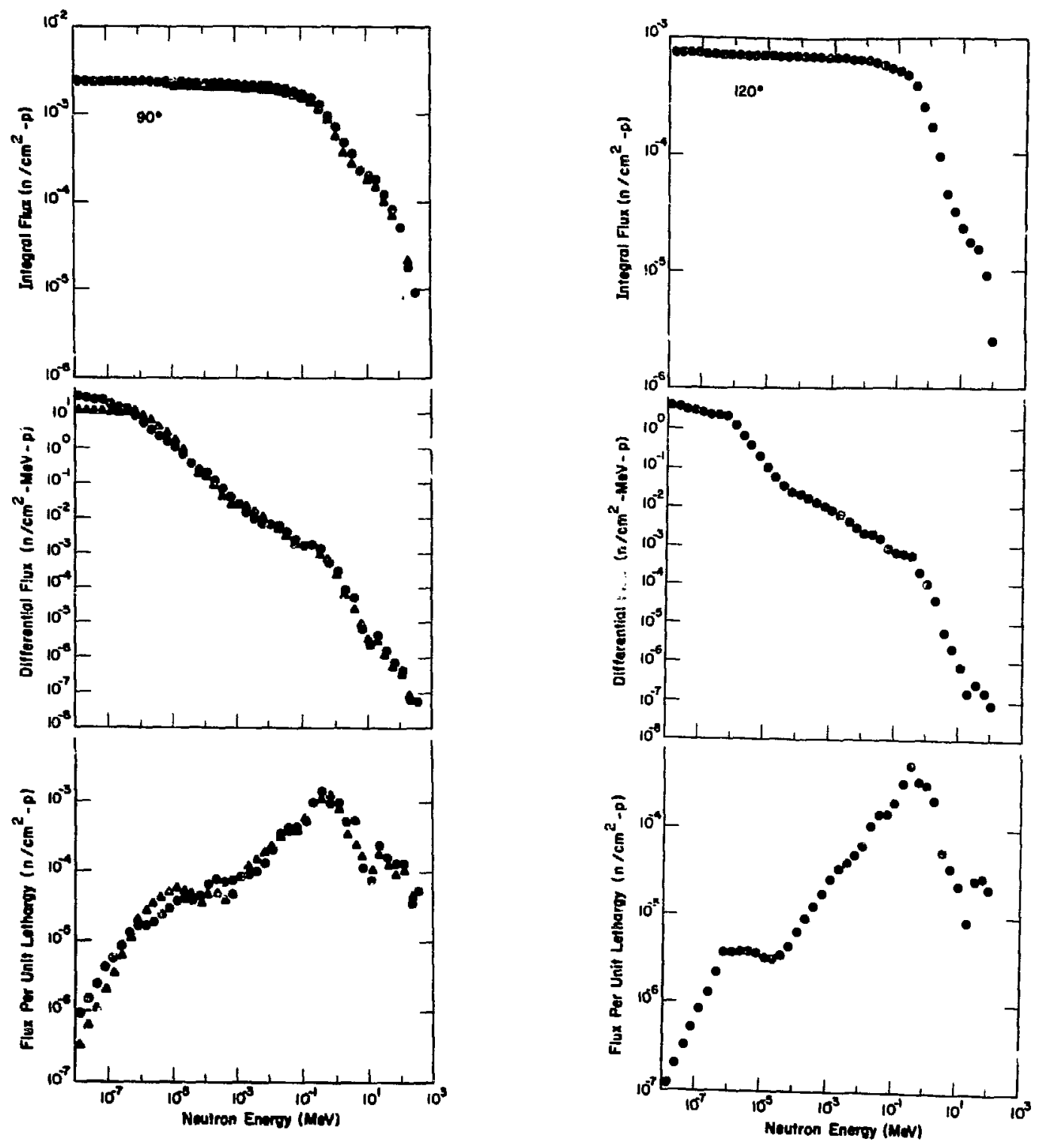

Fig. 6. Calculated neutron flux in the rabbit line for an angie of $90^{\circ}$ from the center of the copper beam stop. $O$ indicate beam stop in $i$ ts extreme downstream position. $\Delta$ indicate extreme upstream position of the beam

Fig. 7. Calculated neutron flux in the rabbit line for an angle of $120^{\circ}$ from the center of the copper beam stop. 


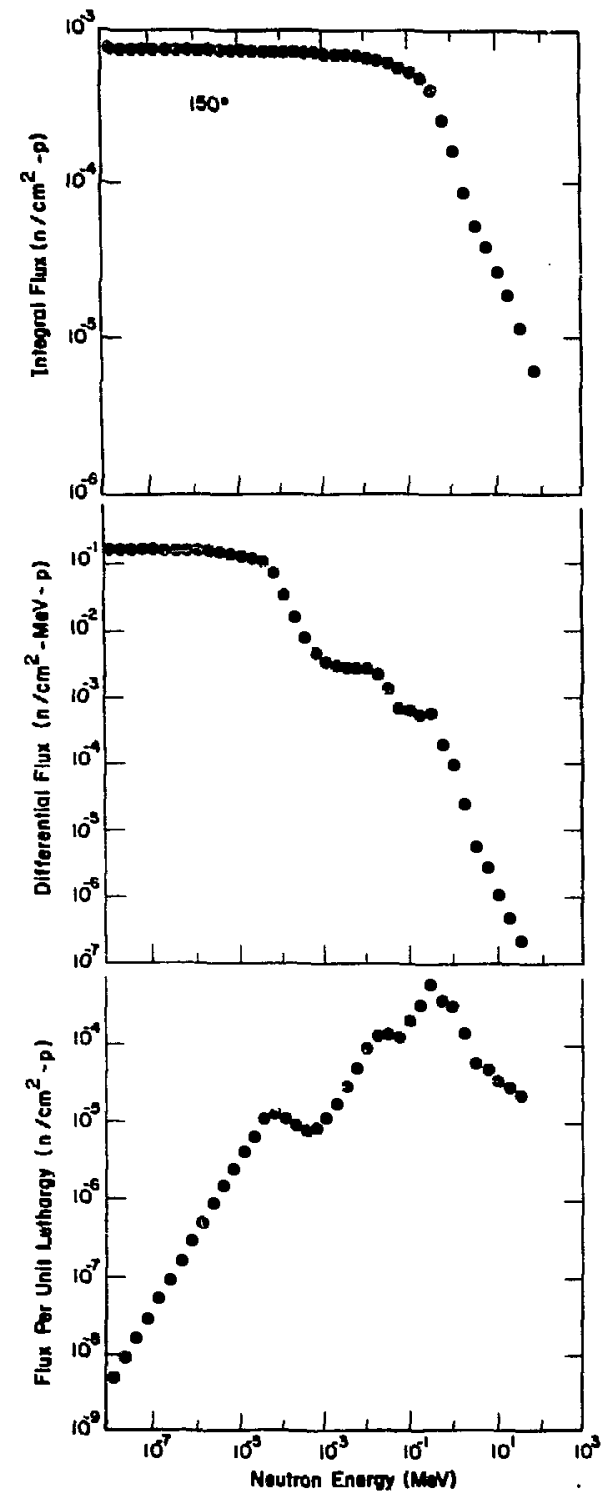

Fig. 8 Calculated neutron flux in the rabbit line for an angle of $150^{\circ}$ from the center of the copper beam stop. 